BMJ Open

Diabetes

Research

\& Care

\title{
Metabolomic, hormonal and physiological responses to hypoglycemia versus euglycemia during exercise in adults with type 1 diabetes
}

\author{
Olivia McCarthy (1) , ${ }^{1}$ Jason Pitt, ${ }^{1}$ Rachel Churm, ${ }^{1}$ Gareth J Dunseath, ${ }^{2}$ \\ Charlotte Jones, ${ }^{2}$ Lia Bally (1) , ${ }^{3}$ Christos T Nakas, ${ }^{4,5}$ Rachel Deere, ${ }^{6}$ \\ Max L Eckstein, ${ }^{7}$ Stephen C Bain, ${ }^{2}$ Othmar Moser (1) , ${ }^{7}$ Richard M Bracken ${ }^{1}$
}

To cite: McCarthy 0 , Pitt J, Churm R, et al. Metabolomic, hormonal and physiological responses to hypoglycemia versus euglycemia during exercise in adults with type 1 diabetes. BMJ Open Diab Res Care 2020;8:e001577. doi:10.1136/ bmjdrc-2020-001577

Received 16 May 2020

Revised 22 July 2020

Accepted 19 August 2020

\section{Check for updates}

(c) Author(s) (or their employer(s)) 2020. Re-use permitted under CC BY-NC. No commercial re-use. See rights and permissions. Published by BMJ.

For numbered affiliations see end of article.

Correspondence to Dr Olivia McCarthy; olivia.mccarthy@swansea. ac.uk

\section{ABSTRACT}

Introduction This study sought to compare the metabolomic, hormonal and physiological responses to hypoglycemia versus euglycemia during exercise in adults with type 1 diabetes (T1D).

Research design and methods Thirteen individuals with T1D (hemoglobin; $7.0 \% \pm 1.3 \%$ (52.6 $\pm 13.9 \mathrm{mmol} / \mathrm{mol})$, age; $36 \pm 15$ years, duration diabetes; $15 \pm 12$ years) performed a maximum of 45 min submaximal exercise $\left(60 \% \pm 6 \% \dot{\mathrm{V}} 0_{2 \max }\right)$. Retrospectively identified exercise sessions that ended in hypoglycemia ((HypoEx) blood glucose $(\mathrm{BG}) \leq 3.9 \mathrm{mmol} / \mathrm{L})$ were compared against a participant-matched euglycemic condition ((EuEx) $B G \geq 4.0, B G \leq 10.0 \mathrm{mmol} / \mathrm{L})$. Samples were compared for detailed physiological and hormonal parameters as well as metabolically profiled via large scale targeted ultrahigh-performance liquid chromatography coupled to tandem mass spectrometry. Data were assessed using univariate and multivariate analysis techniques with false discovery rate adjustment. Significant results were considered at $p \leq 0.05$.

Results Cardiorespiratory and counterregulatory hormone responses, whole-body fuel use and perception of fatigue during exercise were similar under conditions of hypoglycemia and euglycemia (BG $3.5 \pm 0.3$ vs $5.8 \pm 1.1$ $\mathrm{mmol} / \mathrm{L}$, respectively $\mathrm{p}<0.001$ ). HypoEx was associated with greater adenosine salvage pathway activity (5'-methylthioadenosine, $p=0.023$ and higher cysteine and methionine metabolism), increased utilization of glucogenic amino acids (glutamine, $p=0.021$, alanine, aspartate and glutamate metabolism and homoserine/threonine, $\mathrm{p}=0.045$ ) and evidence of enhanced $\beta$-oxidation (lower carnitine $p<0.001$, higher long-chain acylcarnitines). Conclusions Exposure to acute hypoglycemia during exercise potentiates alterations in subclinical indices of metabolic stress at the level of the metabolome. However, the physiological responses induced by dynamic physical exercise may mask the symptomatic recognition of mild hypoglycemia during exercise in people with T1D, a potential clinical safety concern that reinforces the need for diligent glucose management.

Trial registration number DRKS00013509.

\section{INTRODUCTION}

Despite the benefits of regular physical exercise in aiding long-term glycemia control, ${ }^{1}$ the

\section{Significance of this study}

What is already known about this subject?

- Physical exercise-related hypoglycemia is a major clinical concern in the glycemic management of people with type 1 diabetes (T1D).

- Although our pathophysiological understanding of hypoglycemia at rest is well established, less is known about the impact of hypoglycemia on physiological, counterregulatory hormone, perceptual effort and bioenergetic responses to dynamic physical exercise in individuals with $\mathrm{T1D}$.

What are the new findings?

- Under laboratory-controlled conditions, the physiological, hormonal and metabolomic responses of participants who experienced hypoglycemia while performing aerobic exercise were compared with a euglycemic exercising condition.

- Combined with detailed physiological monitoring during exercise, a targeted ultra-high-performance liquid chromatography tandem mass spectrometry approach in the present study has provided new and additive insights of the physiological and bioenergetic responses to exercise under conditions of hypoglycemia and euglycemia in people with T1D.

- Physiological and counterregulatory hormone responses, whole-body fuel use and perception of fatigue to exercise were similar under conditions of hypoglycemia and euglycemia; however, mild hypoglycemia during exercise was associated with signs of intracellular imbalance and an increased reliance on energy pathways involved in purine, lipid and amino acid metabolism.

acute management of glucose during exercise is challenging for those with T1D. The heightened metabolic demands of exercising skeletal muscle can potentiate increases in tissue glucose uptake through mechanisms independent of, but additive to, insulin. ${ }^{2}{ }^{3}$ So to avoid disproportions in the rate of glucose disappearance with the rate of appearance, 


\section{Significance of this study}

How might these results change the focus of research or clinical practice?

- Hypoglycemia during exercise potentiates alterations in subclinical indices of metabolic stress at the level of the metabolome. However, the physiological responses induced by acute dynamic exercise may mask the symptomatic recognition of hypoglycemia during exercise in people with $\mathrm{T1D}$, a potential clinical safety concern that reinforces the need for diligent glucose management.

the body initiates a complex neuroendocrine interplay consisting of a decrement in insulin secretion (to suppress endogenous glucose utilization (EGU) via inhibition of glycogen phosphorylase) opposed by increments in the secretion of glucagon and the catecholamines (which aid endogenous glucose production (EGP) via glycogenolysis and gluconeogenesis). ${ }^{45}$ However, notwithstanding an inability to autoregulate exogenously administered insulin concentrations, the hemodynamic alterations induced by aerobic exercise enhance the mobilization of insulin from the subcutaneous depot. ${ }^{67}$ Hence, the synergistic effects of insulin and exercise in enhancing EGU, ${ }^{8}$ yet suppressing EGP, ${ }^{9}$ predispose exercise-related hypoglycemia in T1D. ${ }^{1011}$

Although several diabetes-specific recommendations for safe performance of physical exercise exist, ${ }^{12}{ }^{13}$ the glycemic response to a given exercise session is highly variable in people with $\mathrm{T} 1 \mathrm{D},{ }^{14}$ thus predicting an expected glucose trend is difficult. The bioenergetic requirements of exercise are highly dependent on its relative intensity and endogenous fuel availability. During the initial stages of submaximal exercise, intramuscular glycogen serves as the prevailing source of energy, with a growing reliance on bloodborne glucose delivered from hepatic glycogenolysis and gluconeogenesis. However, in addition to attenuated glucose counterregulation, ${ }^{15-18}$ individuals with T1D also demonstrate diminished hepatic glycogenolysis during both hypoglycemia ${ }^{17}$ and exercise, ${ }^{19}$ with a supplementary reliance on gluconeogenesis to support EGP. $^{19}$ These aberrations in substrate mobilization may contribute to the aberrant bioenergetic responses to exercise commonly observed in those with T1D. ${ }^{20} 21$

Due to the number of determinants involved in the control of fuel mobilization and metabolism during exercise in people with T1D, assessing the metabolic response to exercise is undoubtedly complex. Although the influence of hyperinsulinemia, ${ }^{8}$ hyperglycemia ${ }^{22}$ and exercise modality ${ }^{23}$ on the metabolic responses to exercise in T1D have recently been investigated, less work has explored the potential implications of acute hypoglycemia during exercise on aspects of energy metabolism in people with T1D, not least because of the practical constraints of safe sample obtention. Given the importance of glucose as a major fuel to support exercising muscle tissue, hypoglycemia may significantly alter the rate and energetic means by which ATP is regenerated during exercise, particularly if combined with inadequate glucose counterregulation. Hence, alongside neurohormonal and physiological analyses, the integration of metabolic profiling techniques that provide insight of energy pathway activity, may advance our current understanding of the metabolic responses to both exercise and hypoglycemia in T1D, and help inform appropriate treatment decisions in clinical care.

\section{Aim}

To compare the metabolomic, hormonal and physiological responses to hypoglycemia versus euglycemia during exercise in adults with type 1 diabetes (T1D).

\section{RESEARCH DESIGN AND METHODS \\ Study design}

This exploratory study involved a retrospective analysis of a single-centre, randomized, open-label, four-period crossover clinical trial consisting of 23 hours inpatient phases with a 45 min evening bout of semi-recumbent cycling at $60 \pm 6 \% \dot{\mathrm{VO}}_{2 \max }$. In the hour before and after exercise, participants administered either a full $(100 \%)$ or reduced $(50 \%)$ dose of individualized bolus insulin aspart alongside identical carbohydrate (CHO)-rich meals $(1 \mathrm{~g} / \mathrm{CHO} / \mathrm{kg} / \mathrm{bm})$. Venous-derived samples were taken hourly leading into cycling, as well as immediately postexercise, then throughout the remaining 14-hour inpatient stay, including nocturnal hours. Trial day hypoglycemia as confirmed by a venous blood glucose (BG) value of $\leq 3.9 \mathrm{mmol} / \mathrm{L}$. All participants were using unblinded interstitial glucose monitoring devices (Freestyle Libre, Abbott, Lake Bluff, Illinois, USA).

\section{Inclusion and exclusion criteria}

Main inclusion criteria were: diagnosis of T1D for $\geq 12$ months; age 18-65 years (both inclusive); body mass index of 18.0-29.4 kg.m²; use of multiple daily injections of insulin for $\geq 12$ months; body mass-specific peak oxygen uptake of $\geq 20 \mathrm{~mL} / \mathrm{kg} / \mathrm{min}$ and a status of being physically active as assessed by the International Physical Activity Questionnaire Short Form. Main exclusion criteria were: presence of a life-threatening disease (ie, proliferative retinopathy or maculopathy, severe neuropathy), recurrent severe hypoglycemia ( $>1$ severe hypoglycemia event during the previous 12 months), hypoglycemia unawareness, hospitalization for diabetic ketoacidosis during the previous 6 months, any other condition that would interfere with trial participation or evaluation of results as judged by the investigators.

\section{Trial design}

As part of a retrospective design using a same-person comparison, the postexercise $B G$ value from each of the four crossover experimental arms was used to identify participants whom on one occasion ended exercise while hypoglycemic ((HypoEx) BG $\leq 3.9 \mathrm{mmol} / \mathrm{L})$, and on one of the other three trials finished exercise while euglycemic ((EuEx) BG $\geq 4.0, \mathrm{BG} \leq 10.0 \mathrm{mmol} / \mathrm{L}){ }^{24}$ The first 
selection criteria for the comparative euglycemic sample was that which was matched in the pre-exercise bolus insulin dosing manipulation, that is, the PreEx100\%PostEx $100 \%$ arm was paired with the PreEx100\%PostEx $50 \%$ arm, while the PreEx50\%-PostEx $50 \%$ arm was paired with the PreEx50\%-PostEx100\% arm. Failing that, the arm with the highest $\mathrm{BG}$ value still within the euglycemic range was selected over a trial that was closer to the hypoglycemic threshold. Of a possible 16 individuals who completed all 4 experimental visits, the data from 13 individuals were suitable for inclusion in this exploratory study.

\section{Moderate-intensity continuous exercise testing protocol and hypoglycemia identification}

The evening (17:00) exercise test consisted of maximum of $45 \mathrm{~min}$ ( $3 \mathrm{~min}$ warm-up at $20 \mathrm{~W}, 42 \mathrm{~min}$ at target workload) of continuous cycling on a semi-recumbent ergometer (Corival Recumbent, Lode, The Netherlands) at $60 \pm 6 \% \dot{\mathrm{V}}_{2 \max }$. The target workload intensity was computed as the mid-point between the first and second lactate turn points. Participants were instructed to maintain a stable pedal cadence between 65 and 75 revolutions per minute (RPM) against a fixed workload. During exercise, heart rate (HR (s410, Polar, Finland)), respiratory (METAMAX 3B; Cortex Biophysik GmbH, Germany) and power metrics were collected in an integrative nature and measured continuously. The $60 \mathrm{~s}$ averages of $5 \mathrm{~s}$ intervals were used to determine gas exchange variables rates at rest, following the warm-up, at each 6 min interval over the target workload, and immediately prior to exercise cessation. Rates of $\mathrm{CHO}$ and lipid oxidation were determined via indirect calorimetry using the stoichiometric equations suitable for moderate-intensity exercise as described previously. ${ }^{25} \mathrm{O}_{2}$ pulse was calculated as the $\mathrm{O}_{2} / \mathrm{HR}$ ratio. In addition to a $4 \mu \mathrm{L}$ fingertip BG measurement (used for safety purposes and assessed immediately via the built-in glucometer (Freestyle libre)), a $20 \mu \mathrm{L}$ capillary blood sample was collected every 6 min from the right earlobe and used for within exercise glucose and lactate analysis via the fully enzymaticamperometric method ((FEA) Biosen C-Line, EKF Diagnostic, Germany). Rate of perceived exertion (RPE (Borg scale)) was also captured every 6 min. If fingertip BG reached $\leq 3.9 \mathrm{mmol} / \mathrm{L}$ prior to completion of the full $45 \mathrm{~min}$ test protocol, exercise testing was terminated immediately. At this point, a venous blood sample was drawn instantly and measured for BG and lactate (BLa). In the event of hypoglycemia, participants received a standardized CHO gel as a rescue aid (Glucogel, BBI Healthcare, UK) and were monitored to ensure recovery to euglycemic levels.

\section{Venous-derived biomarker analysis}

The Randox Daytona Plus RX series analyzer (Randox Laboratories, UK) was used for in vitro determination of $\beta$-hydroxybutyrate ( $(\beta$-OHB) RB4067). ELISAs were used for the quantification of plasma glucagon (DGCG0,
R\&D Systems, Minneapolis, USA) and catecholamines (epinephrine (EPI) and norepinephrine (NE) ECT31K02, Eagle Biosciences, New Hampshire, USA)). BG and BLa were analyzed via FEA.

\section{Metabolomic sample preparation}

To assess the metabolomic responses to exercise-induced hypoglycemia, a metabolic profile of EDTA plasma was acquired by a targeted ultra-high-performance liquid chromatography (reversed phase) coupled to tandem mass spectrometry (UHPLC-MS/MS) approach. Samples were thawed, vortexed and $2 \times 100 \mu \mathrm{L}$ plasma were transferred into two 96 -well plates $(1 \times$ for large-scale targeted MV026, 1× back-up). The protein precipitation and metabolite extraction were conducted with addition of $300 \mu \mathrm{L}$ of cold acetonitrile:ethanol 1:1 (volume/volume) containing internal standards. After vortexing, $250 \mu \mathrm{L}$ of clear supernatant was transferred into a new 96 -well plate and dried using speed vacuum. On the day of analysis, samples were reconstituted in $200 \mu \mathrm{L}$ of $10 \%$ methanol in water containing $1 \mu \mathrm{g} / \mathrm{mL}$ chlorpropamide (dilution factor 1:2), vortexed, sonicated for $4 \mathrm{~min}$ in a water bath and centrifuged at $4400 \mathrm{~g}$ for $20 \mathrm{~min}$ at $4^{\circ} \mathrm{C}$. The supernatants $(150 \mu \mathrm{L})$ were transferred into a new 96-well plate and a quality control was prepared pooling $5 \mu \mathrm{L}$ of each sample. An additional $2 \times 100 \mu \mathrm{L}$ of serum were extracted and the dried sample was reconstituted in $200 \mu \mathrm{L}$ (1:2) and $400 \mu \mathrm{L}$ (1:4) prior to analysis.

\section{UHPLC-MS/MS analysis}

Samples were analyzed in a randomized block design order on a triple quadrupole mass spectrometer (Xevo TQ-S, Waters) coupled to an UPLC Acquity I-Class system (Waters) by reversed-phase chromatography. Samples were first analyzed in negative electrospray ionization (ESI) mode followed by positive ESI mode and finally by selected reaction monitoring.

\section{Statistical analysis}

All statistical analyses were carried out using IBM SPSS V.26.0 (IBM, Armonk, New York, USA) and R V.3.6.1 (The R Foundation for Statistical Computing, Vienna, Austria). Forward stepwise linear regression was used to explore relationships between trial arm glycemia and its metabolic marker. End of exercise data were assessed by means of univariate analysis with exercise duration, preexercise insulin dose amounts (inclusive of basal and bolus amounts), insulin arm (full or reduced dose) and the paring factor accounted for as covariates in the model. False data recovery adjustment was used for the correction of multiple testing. A repeated measures analysis of variance on three levels was conducted with Bonferronicorrected pairwise comparisons used to examine time effects. Pearson's correlations were used to explore associations between metabolomic, and hormonal data based on glycemic grouping. Non-parametric equivalents were used when necessary. $\mathrm{P}$ values of $\leq 0.05$ were considered to be statistically significant. The raw mass spectrometric 


\begin{tabular}{|c|c|}
\hline Characteristic & $\mathrm{n}=13$ \\
\hline Gender M, F (n) & 11 to 2 \\
\hline Age (years) & $36 \pm 15$ \\
\hline BMI (kg. $\left.\mathrm{m}^{2}\right)$ & $26.8 \pm 3.3$ \\
\hline $\mathrm{HbA}_{1 \mathrm{c}}(\%)$ & $7.0 \pm 1.3$ \\
\hline $\mathrm{HbA}_{1 \mathrm{c}}(\mathrm{mmol} / \mathrm{mol})$ & $52.6 \pm 13.9$ \\
\hline Diabetes duration (years) & $15.3 \pm 12.1$ \\
\hline Prestudy TDD (U/kg/bm) & $0.6 \pm 0.3$ \\
\hline Prestudy TDBD (U/kg/bm) & $0.4 \pm 0.2$ \\
\hline$\dot{\mathrm{V}} \mathrm{O}_{2 \max }(\mathrm{mL} / \mathrm{kg} / \mathrm{min})$ & $39.2 \pm 11.0$ \\
\hline
\end{tabular}

Data are presented as mean \pm SD.

$\mathrm{bm}$, body mass; $\mathrm{BMI}$, body mass index; $\mathrm{F}$, female; $\mathrm{HbA}$, hemoglobin $A_{1 c}$; $M$, male; $n$, number of participants; TDBD, total daily basal insulin dose; TDD, total daily insulin dose (inclusive of basal and bolus amounts); $\mathrm{U}$, units of insulin; $\mathrm{VO} 2_{\text {max }}$, maximum volume of inhaled oxygen.

data were processed with Skyline (V.19.1, MacCoss Lab). The peak area data of positive and negative ionization mode were combined. The prominence of circulating of metabolites in relation to glycemic grouping was assessed via cluster analysis, while partial least squares-discriminant analysis was performed to map the inter-relationships between all input-output data. Cross-tabulation analysis was used to identify estimated risk ratios (ERR) between nominal variables, with Fisher's exact test used to report significance.

\section{RESULTS}

The baseline characteristics of study participants are detailed in table 1 .

There were no differences between trials in the total daily dose of insulin ((inclusive of basal and bolus doses) HypoEx $44 \pm 23$ vs EuEx $45 \pm 23 \mathrm{U}, \mathrm{p}=0.395$ ) or total CHO intake (HypoEx 243 \pm 38 vs EuEx 242 \pm 41 g, p=0.961) taken in the hours leading into exercise. Trials were also identical in the amount of bolus insulin taken in the immediate hour prior to exercise (HypoEx; $4 \pm 2$ (31\% dose reduction) vs EuEx; $4 \pm 3 \mathrm{U}$ (38\% dose reduction), $\mathrm{p}=0.674)$, which was injected alongside the consumption of identical CHO rich meals $(1 \mathrm{~g} / \mathrm{CHO} / \mathrm{kg} / \mathrm{bm})$. BG concentrations were similar at baseline (HypoEx 5.8 \pm 1.3 vs EuEx $6.6 \pm 1.8 \mathrm{mmol} / \mathrm{L}, \mathrm{p}=0.206)$ and immediately pre-exercise (HypoEx 7.6 \pm 2.3 vs EuEx $9.5 \pm 2.5 \mathrm{mmol} / \mathrm{L}$, $\mathrm{p}=0.058$ ).

\section{Physiological and metabolic responses to acute moderate- intensity continuous exercise}

The physiological responses at rest, as an exercising mean during the target workload, and immediately at the end of exercise are presented in table 2. There were no differences between groups in any physiological parameter in any of the identified timepoints. Hypoglycemia trended towards slightly earlier test termination (HypoEx $38 \pm 8$ vs EuEx $44 \pm 3$ min, $\mathrm{p}=0.05$ ).

\section{Glycemic, metabolic and counterregulatory hormonal responses to exercise}

Table 3A details the change in metabolic and counterregulatory hormonal biomarkers in response to exercise, while table 3B displays the point concentrations in biomarkers immediately postexercise.

End of exercise BG concentrations were significantly lower during HypoEx (table 3, p<0.001). Neither the magnitude of the drop (table 3 ) nor the rate of change (HypoEx $-0.11 \pm 0.06$ vs EuEx $-0.08 \pm 0.05 \mathrm{mmol} / \mathrm{L} / \mathrm{min}$, $\mathrm{p}=0.700)$ in $\mathrm{BG}$ differed between trials. There were no other differences between groups in either the exerciseinduced change (table 3) or end of exercise concentrations (table 3) in metabolic and counterregulatory hormonal biomarkers.

The exercise-induced change in EPI $(r=-0.612$, $\mathrm{p}=0.026)$, NE $(\mathrm{r}=-0.902, \mathrm{p}<0.001)$ and $\beta$-OHB $(\mathrm{r}=-0.685$, $\mathrm{p}=0.010$ ) were all inversely related to the exercise-induced change in BG during hypoglycemia but not euglycemia. Additionally, end of exercise EPI $(r=-0.669, p=0.012)$, $\mathrm{NE}(\mathrm{r}=-0.576, \mathrm{p}=0.039)$, glucagon $(\mathrm{r}=-0.751, \mathrm{p}=0.005)$, $\beta$-OHB $(r=-0.559, p=0.039)$ and BLa $(r=-0.621, p=0.023)$ concentrations were all inversely related to end of exercise BG under conditions of hypoglycemia only.

\section{Metabolomic responses to submaximal exercise}

Figure 1 provides a multipictorial overview of the systemic metabolic changes during exercise under conditions of hypoglycemia and euglycemia in people with T1D using a targeted UHPLC-MS/MS approach. In total, 87 metabolites were detected, 55 in positive ion mode, 27 in negative ion mode and 5 in both modes. Specifically, the cluster of 1'-methylnicotinamide, L-methionine, citrulline, L-glutamine, pyridoxamine and glucose corresponded to a visible separation between HypoEx and EuEx. Another small cluster was formed by 5'-methyladenosine and cytidine (figure 1B). These results were confirmed from the univariate analysis (figure 1C-E). Further pathway enrichment analysis using the Metaboanalyst platform defined the difference between HypoEx and EuEx to be characterized from cysteine and methionine metabolism as well as alanine, aspartate and glutamate metabolism.

Figure 2A details the correlation-based metabolic network analysis. Variable closer together have high correlations among them while those opposite have high negative correlations. Variables in the outer circle are the most relevant in driving the HypoEx-EuEx separation. Figure 2B displays the discriminating metabolites that differed significantly between groups at the point of exercise cessation.

HypoEx was associated with higher 5'-methylthioadenosine (fold change: $1.468 \pm 0.515,95 \%$ CI 1.262 to 1.674 , $\mathrm{p}=0.023$ ) and L-homoserine/L-threonine (fold change: $1.108 \pm 0.230$, $95 \%$ CI 1.016 to $1.200, p=0.045$ ), but lower L-glutamine (fold change: $0.898 \pm 0.088,95 \%$ CI 0.863 to 
Table 2 The physiological responses to exercise

\begin{tabular}{|c|c|c|c|c|}
\hline Parameter & Rest & Exercise & End of exercise & $\begin{array}{l}\text { End of exercise } p \\
\text { value }\end{array}$ \\
\hline \multicolumn{5}{|l|}{$\dot{\mathrm{V}}_{2}(\mathrm{~L} / \mathrm{min})$} \\
\hline Euglycemia & $0.405 \pm 0.088$ & $1.87 \pm 0.36^{\star}$ & $1.958 \pm 0.420^{\star}$ & \multirow[t]{2}{*}{0.889} \\
\hline Hypoglycemia & $0.343 \pm 0.085$ & $1.85 \pm 0.38^{*}$ & $1.860 \pm 0.400^{*}$ & \\
\hline \multicolumn{5}{|l|}{$\dot{\mathrm{V} C O}{ }_{2}(\mathrm{~L} / \mathrm{min})$} \\
\hline Euglycemia & $0.36 \pm 0.08$ & $1.77 \pm 0.06^{*}$ & $1.85 \pm 0.42^{*}$ & \multirow[t]{2}{*}{0.835} \\
\hline Hypoglycemia & $0.31 \pm 0.07$ & $1.76 \pm 0.36^{\star}$ & $1.75 \pm 0.35^{\star}$ & \\
\hline \multicolumn{5}{|c|}{ Respiratory exchange ratio (L/min) } \\
\hline Euglycemia & $0.90 \pm 0.03$ & $0.95 \pm 0.03^{*}$ & $0.94 \pm 0.03^{*}$ & \multirow[t]{2}{*}{0.954} \\
\hline Hypoglycemia & $0.91 \pm 0.07$ & $0.95 \pm 0.03^{*}$ & $0.94 \pm 0.04^{*}$ & \\
\hline \multicolumn{5}{|c|}{ Carbohydrate oxidation (g/min) } \\
\hline Euglycemia & $0.3 \pm 0.1$ & $1.9 \pm 0.5^{\star}$ & $2.0 \pm 0.6^{*}$ & \multirow[t]{2}{*}{0.786} \\
\hline Hypoglycemia & $0.3 \pm 0.1$ & $1.9 \pm 0.4^{*}$ & $1.9 \pm 0.4^{*}$ & \\
\hline \multicolumn{5}{|c|}{ Lipid oxidation (g/min) } \\
\hline Euglycemia & $0.069 \pm 0.023$ & $0.17 \pm 0.08^{*}$ & $0.185 \pm 0.845^{\star}$ & \multirow[t]{2}{*}{0.811} \\
\hline Hypoglycemia & $0.058 \pm 0.040$ & $0.15 \pm 0.07^{*}$ & $0.189 \pm 0.117^{\star}$ & \\
\hline \multicolumn{5}{|c|}{ Oxygen pulse (mL/beat) } \\
\hline Euglycemia & $0.007 \pm 0.005$ & $0.01 \pm 0.00^{*}$ & $0.01 \pm 0.00^{*}$ & \multirow[t]{2}{*}{0.269} \\
\hline Hypoglycemia & $0.003 \pm 0.005$ & $0.01 \pm 0.00^{*}$ & $0.01 \pm 0.00^{*}$ & \\
\hline \multicolumn{5}{|c|}{ Minute ventilation ( $\mathrm{L} / \mathrm{min})$} \\
\hline Euglycemia & $14.2 \pm 2.4$ & $52.2 \pm 9.2^{*}$ & $55.5 \pm 11.6^{*}$ & \multirow[t]{2}{*}{0.852} \\
\hline Hypoglycemia & $12.3 \pm 2.2$ & $52.1 \pm 9.9^{*}$ & $52.8 \pm 8.9^{*}$ & \\
\hline \multicolumn{5}{|c|}{ Tidal volume (L/min) } \\
\hline Euglycemia & $0.69 \pm 0.16$ & $1.68 \pm 0.41^{*}$ & $1.71 \pm 0.46^{*}$ & \multirow[t]{2}{*}{0.925} \\
\hline Hypoglycemia & $0.66 \pm 0.12$ & $1.74 \pm 0.42^{*}$ & $1.69 \pm 0.45^{\star}$ & \\
\hline \multicolumn{5}{|c|}{ End-tidal oxygen partial pressure $(\mathrm{mm} \mathrm{Hg})$} \\
\hline Euglycemia & $109.60 \pm 2.91$ & $107.42 \pm 3.21^{*}$ & $108.36 \pm 4.16$ & \multirow[t]{2}{*}{0.460} \\
\hline Hypoglycemia & $109.88 \pm 6.15$ & $106.21 \pm 4.24^{*}$ & $106.75 \pm 4.56^{\star}$ & \\
\hline \multicolumn{5}{|c|}{ End-tidal carbon dioxide partial pressure $(\mathrm{mm} \mathrm{Hg})$} \\
\hline Euglycemia & $35.37 \pm 2.59$ & $38.02 \pm 2.69^{\star}$ & $37.01 \pm 3.32$ & \multirow[t]{2}{*}{0.919} \\
\hline Hypoglycemia & $34.25 \pm 3.59$ & $38.23 \pm 3.59^{\star}$ & $37.33 \pm 3.61^{*}$ & \\
\hline \multicolumn{5}{|c|}{ Pedal revolutions per minute (revolutions/min) } \\
\hline Euglycemia & $0 \pm 0$ & $71 \pm 6^{*}$ & $72 \pm 8^{*}$ & \multirow[t]{2}{*}{0.215} \\
\hline Hypoglycemia & $0 \pm 0$ & $68 \pm 7^{*}$ & $66 \pm 10^{*}$ & \\
\hline \multicolumn{5}{|l|}{ Heart rate (bpm) } \\
\hline Euglycemia & $75 \pm 9$ & $136 \pm 13^{*}$ & $142 \pm 14^{*}$ & \multirow[t]{2}{*}{0.438} \\
\hline Hypoglycemia & $77 \pm 13$ & $136 \pm 14^{*}$ & $145 \pm 16^{*}$ & \\
\hline \multicolumn{5}{|c|}{ Rate of perceived exertion (Borg scale) } \\
\hline Euglycemia & $6 \pm 3$ & $11 \pm 2^{*}$ & $12 \pm 3^{*}$ & \multirow[t]{2}{*}{0.150} \\
\hline Hypoglycemia & $6 \pm 0$ & $10 \pm 2^{*}$ & $13 \pm 3^{*}$ & \\
\hline
\end{tabular}

Data are presented as mean $\pm S D(n=13)$. Spirometry data are reported as the $60 \mathrm{~s}$ mean of $5 \mathrm{~s}$ data points over each 6 min time interval.

Data have been treated via univariate analysis with exercise duration, pre-exercise insulin dosing amount (inclusive of all basal and bolus doses) and the insulin dosing arm (ie, full vs reduced dose) included as covariates in the model.

${ }^{*} \mathrm{P} \leq 0.05$ in the comparison with the resting value.

$\mathrm{VCO}_{2}$, volume of exhaled carbon dioxide; $\mathrm{VO}_{2}$, volume of inhaled oxygen.

0.933, $\mathrm{p}=0.021$ ) and L-carnitine (fold change: $0.837 \pm 0.123$, $95 \%$ CI 0.788 to $0.886, \mathrm{p}<0.001$ ) concentrations (figure $2 \mathrm{~B}$ ).

\section{DISCUSSION}

This novel, exploratory study characterized the physiological, pancreatic/counter regulatory hormone and metabolomic responses to euglycemic exercise in adults with T1D and compared them with an exercise session that resulted in hypoglycemia. Combined with detailed physiological monitoring during exercise, integration of an UHPLC-MS/MS platform provided new and additive insights into the influence of hypoglycemia and 
Table 3 End of exercise venous derived biomarker concentrations for the hypoglycemia (HypoEx) versus euglycemia (EuEx) trials

\begin{tabular}{|c|c|c|c|}
\hline \multicolumn{4}{|c|}{ (A) Exercise-induced change in biomarkers } \\
\hline Biomarker & HypoEx & EuEx & $P$ value \\
\hline $\begin{array}{l}\text { Blood glucose } \\
\text { (mmol/L) }\end{array}$ & $-4.1 \pm 2.5^{\star}$ & $-3.6 \pm 2.2^{*}$ & 0.698 \\
\hline $\begin{array}{l}\text { Blood lactate } \\
\text { (mmol/L) }\end{array}$ & $+1.8 \pm 0.0 .9^{\star}$ & $+2.0 \pm 1.8^{*}$ & 0.916 \\
\hline$\beta-\mathrm{OHB}(\mathrm{mmol} / \mathrm{L})$ & $+0.01 \pm 0.01$ & $+0.01 \pm 0.02$ & 0.945 \\
\hline Glucagon (pg/mL) & $+16.8 \pm 64.8$ & $+1.6 \pm 13.0$ & 0.348 \\
\hline EPI (nmol/L) & $+0.05 \pm 0.08$ & $+0.03 \pm 0.12$ & 0.779 \\
\hline $\mathrm{NE}(\mathrm{nmol} / \mathrm{L})$ & $+0.63 \pm 1.11$ & $+0.77 \pm 1.33$ & 0.878 \\
\hline \multicolumn{4}{|c|}{ (B) End of exercise point concentrations in biomarkers } \\
\hline Biomarker & HypoEx & EuEx & $P$ value \\
\hline $\begin{array}{l}\text { Blood glucose } \\
\text { (mmol/L) }\end{array}$ & $3.5 \pm 0.3$ & $5.8 \pm 1.1$ & $<0.001$ \\
\hline Blood lactate $(\mathrm{mmol} / \mathrm{L})$ & $2.7 \pm 0.9$ & $3.0 \pm 1.9$ & 0.812 \\
\hline$\beta-\mathrm{OHB}(\mathrm{mmol} / \mathrm{L})$ & $0.05 \pm 0.01$ & $0.05 \pm 0.02$ & 0.945 \\
\hline Glucagon (pg/mL) & $28.5 \pm 61.04$ & $36.7 \pm 72.1$ & 0.964 \\
\hline EPI (nmol/L) & $0.08 \pm 0.09$ & $0.08 \pm 0.13$ & 0.817 \\
\hline NE (nmol/L) & $1.37 \pm 0.93$ & $1.45 \pm 1.49$ & 0.635 \\
\hline
\end{tabular}

Data are reported as mean $\pm S E M ; n=13$.

Data have been treated via univariate analysis with exercise duration, pre-exercise insulin dosing amount (inclusive of all basal and bolus doses) and the insulin dosing arm (ie, full vs reduced dose) included as covariates in the model.

${ }^{*} \mathrm{P} \leq 0.05$ denotes significant difference compared with the corresponding immediate pre-exercise value.

EPI, epinephrine; Ex, exercise; NE, norepinephrine; $\beta-\mathrm{OHB}$, $\beta$-hydroxybutyrate.

euglycemia during exercise on the activity of diverse metabolic pathways in people with T1D.

Autonomic system activation during exercise, principally an increase in sympathetic activity, and withdrawal of parasympathetic activity, is critical in facilitating stimulation of several cardiovascular and hemodynamic processes aimed at maintaining glucose and oxygen supply to exercising muscle tissue in the face of increasing intracellular energy demands. ${ }^{26}{ }^{27}$ Interestingly, many of the physiological responses to dynamic exercise share similar characteristics with the symptomatic responses to acute hypoglycemia (ie, sympathetic neural activation, sweating, pallor, elevated heart rate, observations of central and peripheral fatigue) ${ }^{28}$ Thus, it could be postulated that the concurrent appearance of two physiological stressors (ie, exercise and hypoglycemia) would produce greater physiological, metabolic and counterregulatory responses than those induced by exercise and/or hypoglycemia per se. However, despite significant differences in the BG values at the point of sample obtention ( 3.5 vs $5.8 \mathrm{mmol} / \mathrm{L}$ ), we found no influence of hypoglycemia on the cardiorespiratory responses, whole-body fuel use or the perception of effort to the same power output.
Hence, it could be possible that for the exercising individual with $\mathrm{T} 1 \mathrm{D}$, the simultaneous occurrence of exercise and hypoglycemia may somewhat hinder the ability to clearly disseminate symptoms that are exclusive to hypoglycemia per se, versus those which are being driven by the physiological strain of dynamic physical exercise per se. Importantly, none of the participants in the present study were hypoglycemic unaware, thus it is intriguing to think of how these responses may have differed in a cohort with such a diagnosis.

The BG value at the end of exercise in hypoglycemic arm fell within the bracket of level 1 hypoglycemia $(\geq 3.0$ to $\leq 3.9 \mathrm{mmo} / \mathrm{L}$ ) in people with T1D. ${ }^{29} 30$ Although this was significantly greater than the equivalent euglycemic value, the glycemic threshold for glucose counterregulation in people with T1D is highly individualized and related to the degree of hypoglycemic awareness. ${ }^{31}$ Strict glycemic control in order to attain target hemoglobin $\mathrm{A}_{1 \mathrm{c}}\left(\mathrm{HbA}_{1 \mathrm{c}}\right)(\sim 7.1 \%)$ can lower the plasma glucose level required to generate a counterregulatory hormone response to concentrations deemed severely hypoglycemic $(\sim 2.6 \mathrm{mmol} / \mathrm{L})$, and hence, diminish patient recognition of moderate hypoglycemia. ${ }^{32}$ Thus, the immediate correction of mild $(\leq 3.9 \mathrm{mmo} / \mathrm{L})$, as opposed to severe $(<3.0 \mathrm{mmol} / \mathrm{L})$, hypoglycemia in the present study may explain the similarities in our neurohormonal data, by virtue of our threshold being set 'too high', particularly given the appreciable glycemic control of our participants $\left(\mathrm{HbA}_{1 \mathrm{c}}\right.$ of $\sim 7.0 \%$ ). This may also explain the observed presence of inverse relationships between $\mathrm{BG}$ and the counterregulatory hormones in the hypoglycemic arm only, emphasizing the growing importance of these biomarkers in aiding EGP as glucose declines towards level 2, but not necessarily level 1, hypoglycemia.

Indeed, we observed no discernible differences in either glucagon or catecholamine concentrations under exercising conditions of hypoglycemia and euglycemia, suggesting a failure of mild hypoglycemia to induce sufficient glucose counterregulation in people with T1D, an established finding within literature. ${ }^{171933}$ The hormonal regulatory effects on gluconeogenesis and glycogenolysis during exercise have been studied extensively, and as per the key principles of glucose counterregulation at rest, a low insulin:glucagon ratio is essential in potentiating EGP to avoid and/or rapidly correct hypoglycemia. ${ }^{34-36}$ In the current study, our participants likely exercised while hyperinsulinemic, having injected an individualized dose of bolus insulin aspart $60 \mathrm{~min}$ prior to cycling that is directly within its peak action $(\sim 31-70 \mathrm{~min}),{ }^{37}$ alongside the consumption of a carbohydrate-rich meal (65\% carbohydrate content), the latter of which prompts the muscle to preferentially use glucose as a source fuel. ${ }^{38}$ This may then explain the comparability between groups in both the rate and magnitude of the drop in glucose (both of which were substantial), the presiding contribution of carbohydrate oxidation to the total energy yield (HypoEx 85\% vs EuEx 83\%, p=0.896), and the inadequate counterregulatory hormonal responses to 
A)

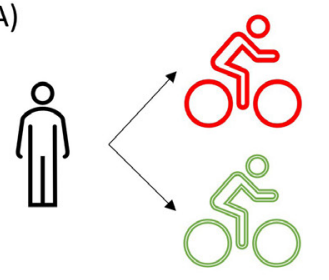

Hypoglycemia



Euglycemia

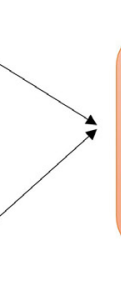

UHPLC-MS/MS

based metabolomic profiling of EDTA

plasma samples

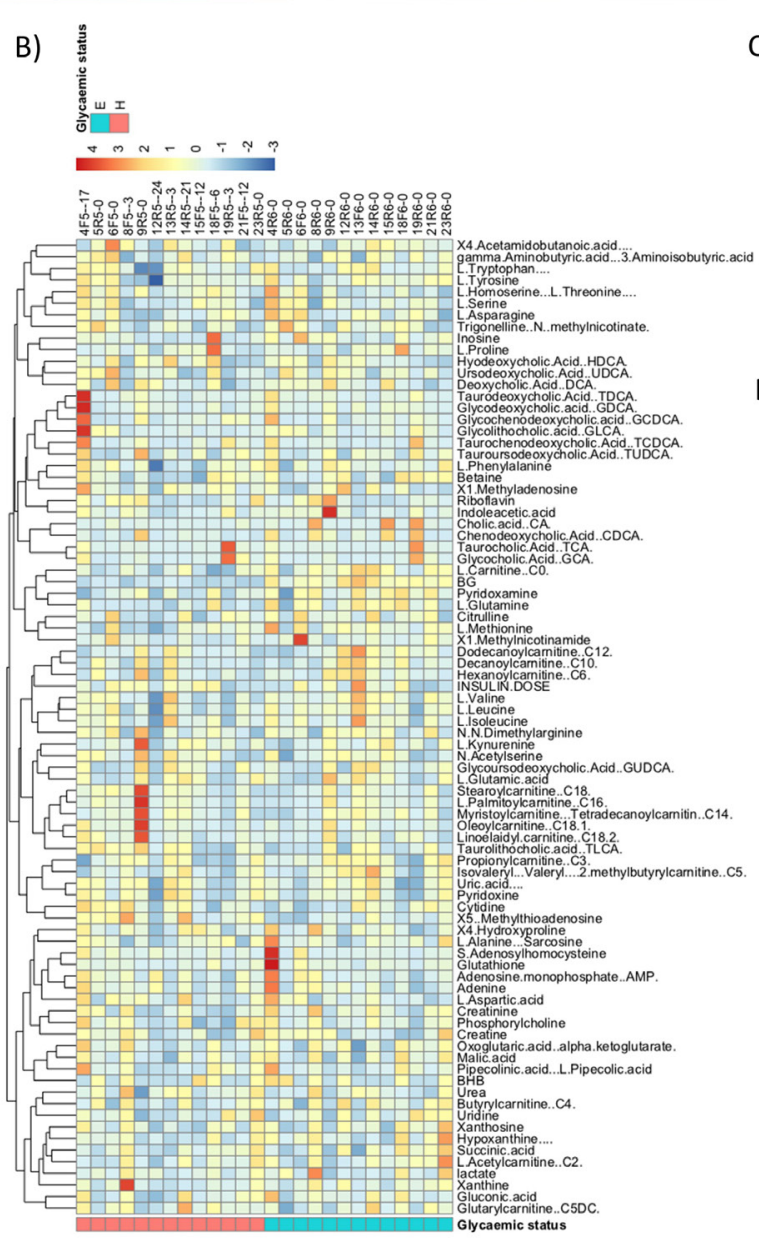

C)

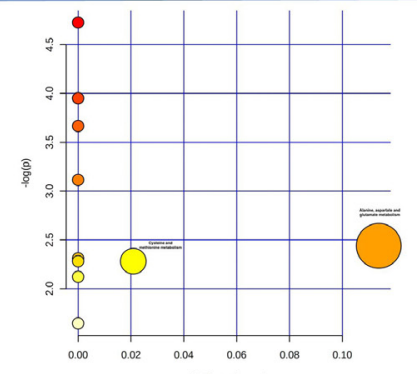

D)
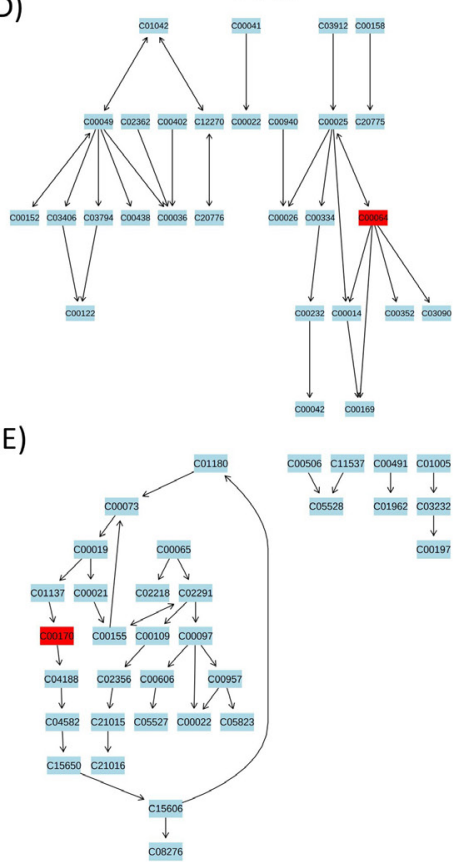

Figure 1 (A) Schematic representation of the experiment. (B) Heat-plot depicting separation quality of hypoglycemia (HypoEx) versus euglycemia (EuEx) at the point of exercise cessation. Some pattern of differences between metabolites for HypoEx versus EuEx is revealed via k-means clustering. Specifically, the cluster of 1'-methylnicotinamide, L-methionine, citrulline, Lglutamine, pyridoxamine and glucose corresponds to a visible separation between hypoglycemia and euglycemia. Another small cluster was formed by 5'-methyladenosine and cytidine. These results were confirmed from the univariate analysis (C-E). Further pathway enrichment analysis using the metaboanalyst platform defined the difference between HypoEx and EuEx to be characterized from cysteine and methionine metabolism as well as alanine, aspartate and glutamate metabolism. UHPLC-MS/ MS, ultra-high-performance liquid chromatography (reversed phase) coupled to tandem mass spectrometry.

both exercise and hypoglycemia as separate, and combined, entities.

Undoubtedly, physical exercise is an intense physiological and metabolic stress and requires the integration of multiple organ systems to coordinate the utilization of intracellular and extracellular energy stores to support ATP resynthesis. ${ }^{39}$ Thus, the use of metabolomics in the present study provided further detailed insights of the metabolic responses to exercise under conditions of hypoglycemia and euglycemia in people with T1D. The elevated concentrations of metabolites involved in purine metabolism during hypoglycemia ((figure 1B), particularly those involved in the salvage pathway, ie, 5 '-methylthioadenosine (figure 2B)) imply a higher rate of intracellular ATP turnover. ${ }^{40}$ MTA is rapidly metabolized by 5 '-methythioadenosine phosphorylase to yield adenine and 5-methylthioribose-1-phosphate (MTR1P). MTR1P is subsequently converted to methionine while the adenine molecule is salvaged to replenish the AMP and ATP pools. ${ }^{42}$ Hypoglycemia during exercise was also associated with significant decrements in plasma levels of carnitine and visual increases in clusters of several longchain (C14-C18) acylcarnitines, suggesting enhanced $\beta$-oxidation. Carnitine is responsible for the translocation 
A)

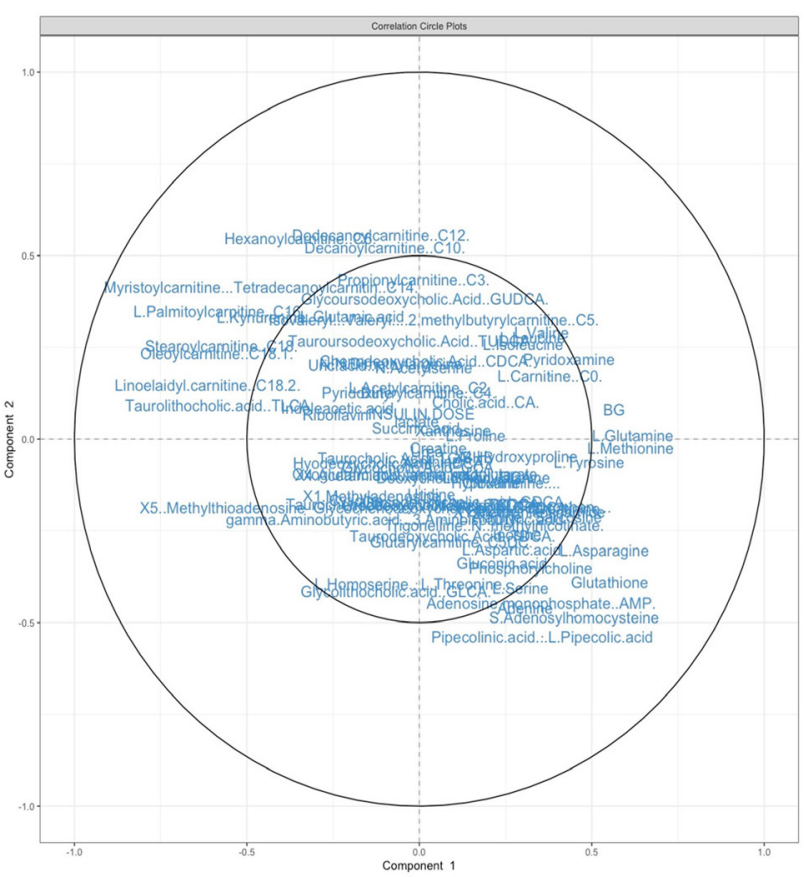

B)

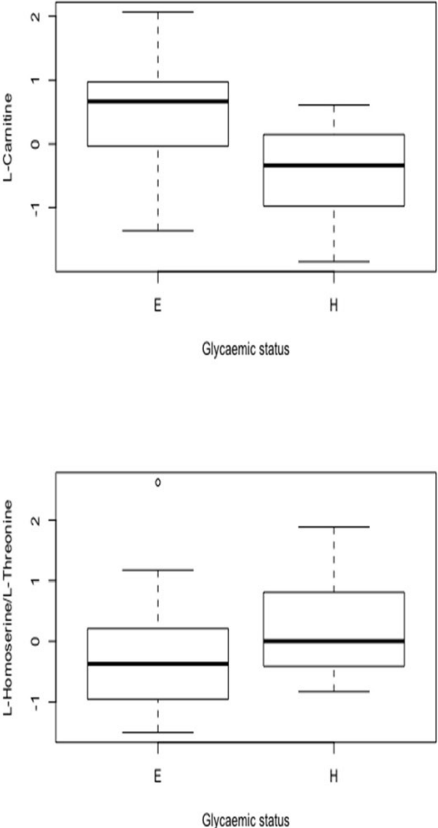

Figure 2 Results from multivariate analysis via principle component analysis (A) and box plots of significant metabolites (B) revealed via univariate analyses based on standard linear modeling. E, euglycemia; $\mathrm{H}$, hypoglycemia.

of long-chain acyl coenzyme A (CoA) (derived from circulating lipids) into the mitochondrial matrix where they undergo oxidation to form acetyl CoA to enter the tricarboxylic acid (TCA) cycle for energy generation. ${ }^{43}$ Finally, during in-exercise hypoglycemia we observed lower concentrations of various glucogenic amino acids (particularly methionine and glutamine), which can be biologically degraded to form intermediates of the TCA cycle, and latterly used for gluconeogenesis. These findings were supported by our pathway analysis which detailed higher alanine, aspartate and glutamate metabolism as well as cysteine and methionine metabolism, indicating higher amino acid metabolism during hypoglycemia. The cysteine produced via methionine metabolism can be used for the biosynthesis of glutathione, a major antioxidant involved in the direct scavenge of radical species to counteract oxidative stress. ${ }^{44}$ Hence, our data reaffirm the pro-oxidative effects of hypoglycemia in people with $\mathrm{T} 1 \mathrm{D},{ }^{45}$ but expand these rested findings to a dynamic exercising environment. The metabolomic signature of hypoglycemia during exercise in the present study was similar to that commonly observed in response to energydepleting exercise ${ }^{2346}$ and/or nutritional deprivation, that is, fasting. ${ }^{47} 48$ Taken collectively, hypoglycemia during exercise was associated with signs of intracellular imbalance and a consequential increase in the activity of energy pathways involved in purine, lipid and amino metabolism.

Although individual metabolites may function as bioactive effectors, their upstream biochemical pathways are often tightly orchestrated by hormonal shifts. ${ }^{47}$ While we did not observe any overt hormonal differences between conditions when assessed as point concentrations, there was a trend for more pronounced glucagon (HypoEx; $+144 \%$ vs EuEx $+4 \%)$ and epinephrine (HypoEx; $+195 \%$ vs EuEx $+71 \%$ ) responses to exercise in the hypoglycemic arm. Glucagon and epinephrine promote EGP via glycogenolysis, gluconeogenesis and lipolysis (the latter via the hydrolysis of triglycerides to yield glycerol (which can be used to support gluconeogenesis) and free fatty acids (which undergo the process described above)). ${ }^{49}$ Thus, it is tempting to speculate that the greater responsiveness of these two hormones during hypoglycemia, may at least partly, explain the divergence in our metabolomic observations, although this hypothesis warrants future exploration. On reflection, it would seem as though while the exposure to acute, level 1 hypoglycemia during exercise may not have been of sufficient duration nor depth to have provoked a potent enough hormonal response, it did appear to potentiate alterations in subclinical indices of metabolic stress at the level of the metabolome.

The high carbohydrate feeding plan leading into exercise in our study the provides reasonable confidence in stating the unlikeliness of significant glycogen depletion over the 45 min submaximal exercise test. It is then interesting to note that hypoglycemia led to an increased utilization of non-carbohydrate containing precursor molecules involved in gluconeogenesis. Previous research has documented attenuated rates of hepatic glycogenolysis during both hypoglycemia ${ }^{17}$ and exercise ${ }^{19}$ in people with T1D, a deficit that is supplemented by excessive rates of hepatic gluconeogenesis. ${ }^{19}$ These alterations in fuel mobilization are believed contributory to the increased risk of recurrent hypoglycemia and hypoglycemiaassociated autonomic failure (HAAF) commonly observed in T1D. This led us to perform subanalysis on 
our data to assess the potential risk of recurrent events. We found that antecedent exercise-induced hypoglycemia was associated with a 1.5-fold increase in the risk of recurrent hypoglycemia in the acute ( 6 hours) postexercise period (HypoEx 5 events vs EuEx 1 event, ERR; 1.5 (95\% CI 0.959 to 2.370 ), $\mathrm{p}=0.06$ ). This was corroborated by a noticeable increase in the amount of time spent in the interstitial-derived hypoglycemic range leading into and throughout the nocturnal hours (HypoEx 2.5 $\pm 4.6 \%$ vs EuEx $0.2 \% \pm 0.5 \%, \mathrm{p}=0.07$ ), findings with clear clinical relevance. It is well established that antecedent hypoglycemia can attenuate the counterregulatory mechanisms against a subsequent event, ${ }^{50} 51$ thereby exposing the individual to a vicious cycle of recurrent hypoglycemiathe classic features of HAAF. The concept of HAAF has recently been expanded to an exercise component (EAAF), with the acknowledgment that antecedent exercise itself may impair the counterregulatory defenses to subsequent hypoglycemia. ${ }^{52}{ }^{53}$ Hence, using a model during which exercise and hypoglycemia occurred simultaneously, our current findings underline the escalation in the risk of recurrent hypoglycemia when the principles of HAAF and EAAF are combined, reaffirming the importance of diligent glucose monitoring around exercise in people with T1D.

\section{Study strengths, limitations and future recommendations}

The application of a validated UHPLC-MS/MS metabolomic profiling platform, combined with counterregulatory hormonal and physiological analyses under controlled laboratory conditions has provided novel insights in the provision and partition of fuel during exercise under conditions of both level 1 hypoglycemia and euglycemia in people with T1D. However, in addition to a small sample size, the homogeneity of study participants including the predominately male gender split, the appreciable glycemic control and the immediate correction of hypoglycemia at a high threshold are important points for consideration when interpreting findings. Further work is needed to deepen our understanding of the influence of hypoglycemia during exercise on various aspects of human physiology in people with T1D.

\section{CONCLUSION}

Exposure to acute hypoglycemia during exercise potentiates alterations in subclinical indices of metabolic stress at the level of the metabolome. However, the physiological responses induced by dynamic physical exercise may mask the symptomatic recognition of mild hypoglycemia during exercise in people with T1D, a potential clinical safety concern that emphasizes the need for increased attention to glucose management.

\footnotetext{
Author affiliations

${ }^{1}$ Applied Sport, Technology, Exercise and Medicine Research Centre (A-STEM),

Swansea University College of Engineering, Swansea, UK

${ }^{2}$ Diabetes Research Group, Swansea University Medical School, Swansea, UK
}

${ }^{3}$ Department of Diabetes, Endocrinology, Nutritional Medicine and Metabolism, Inselspital University Hospital Bern, Bern, Switzerland

${ }^{4}$ Laboratory of Biometry, University of Thessaly, Volos, Thessaly, Greece

${ }^{5}$ Department of Clinical Chemistry, Inselspital, Bern University Hospital, University of Bern, Bern, Switzerland

${ }^{6}$ Department of Health, University of Bath, Bath, UK

${ }^{7}$ Department of Internal Medicine, Medical University of Graz, Graz, Austria

Acknowledgements The authors would like to thank the participants for their significant time commitments and adherence to the study protocol. The authors would also like to thank the clinical metabolomics facility at Inselspital, Universitätsspital Bern, for their invaluable contributions to the analysis of these data.

Contributors OMcC, OM, RD, MLE, JP and RMB were responsible for data collection. RC, GJD and CJ performed laboratory-based hormonal and metabolic data analysis. OMcC, CTN, LB and RMB were responsible for data interpretation and statistical analyses. OMcC and RMB wrote the manuscript. SCB and RMB were the chief and principle investigators of the study. SCB provided medical oversight for the study. RMB wrote and secured funding for the study. All coauthors contributed to feedback and revisions for the final manuscript.

Funding This study was funded by Novo Nordisk UK as part of an ISS.

Competing interests None declared.

Patient consent for publication Not required.

Ethics approval The study was approved by both the national research ethics committee (16/WA/0394) and the local health authority (EudraCT number: 2017004774-34; UTN: U1111-1174-6676). The study was carried out in accordance with the 1964 Declaration of Helsinki and the International Conference on Harmonisation Good Clinical Practice (ICH GCP E6 guidelines). All participants were provided with a full written and verbal description of the study and gave informed consent prior to taking part.

Provenance and peer review Not commissioned; externally peer reviewed.

Data availability statement № data are available.

Open access This is an open access article distributed in accordance with the Creative Commons Attribution Non Commercial (CC BY-NC 4.0) license, which permits others to distribute, remix, adapt, build upon this work non-commercially, and license their derivative works on different terms, provided the original work is properly cited, appropriate credit is given, any changes made indicated, and the use is non-commercial. See: http://creativecommons.org/licenses/by-nc/4.0/.

\section{ORCID iDs}

Olivia McCarthy http://orcid.org/0000-0001-6971-611X

Lia Bally http://orcid.org/0000-0003-1993-7672

Othmar Moser http://orcid.org/0000-0002-1661-0685

\section{REFERENCES}

1 Bohn B, Herbst A, Pfeifer M, et al. Impact of physical activity on glycemic control and prevalence of cardiovascular risk factors in adults with type 1 diabetes: a cross-sectional multicenter study of 18,028 patients. Diabetes Care 2015;38:1536-43.

2 Gao J, Ren J, Gulve EA, et al. Additive effect of contractions and insulin on GLUT-4 translocation into the sarcolemma. J App/ Physiol 1994;77:1597-601.

3 DeFronzo RA, Ferrannini E, Sato Y, et al. Synergistic interaction between exercise and insulin on peripheral glucose uptake. J Clin Invest 1981;68:1468-74.

4 Brooks GA, Fahey TD, White TP. Exercise physiology: human bioenergetics and its applications. Exerc Physiol Hum Bioenerg its Appl 1996.

5 Jensen TE, Richter EA. Regulation of glucose and glycogen metabolism during and after exercise. J Physiol 2012;590:1069-76.

6 Fernqvist $\mathrm{E}$, Linde B, Östman J, et al. Effects of physical exercise on insulin absorption in insulin-dependent diabetics. A comparison between human and porcine insulin. Clin Physiol 1986;6:489-97.

7 Ronnemaa T, Koivisto VA. Combined effect of exercise and ambient temperature on insulin absorption and postprandial glycemia in type I patients. Diabetes Care 1988;11:769-73.

8 Chokkalingam K, Tsintzas K, Norton L, et al. Exercise under hyperinsulinaemic conditions increases whole-body glucose disposal without affecting muscle glycogen utilisation in type 1 diabetes. Diabetologia 2007;50:414-21. 
9 Camacho RC, Pencek RR, Lacy DB, et al. Suppression of endogenous glucose production by mild hyperinsulinemia during exercise is determined predominantly by portal venous insulin. Diabetes 2004:53:285-93.

10 Younk LM, Mikeladze M, Tate D, et al. Exercise-related hypoglycemia in diabetes mellitus. Expert Rev Endocrinol Metab 2011;6:93-108.

11 Briscoe VJ, Tate DB, Davis SN. Type 1 diabetes: exercise and hypoglycemia. Appl Physiol Nutr Metab 2007;32:576-82.

12 Riddell MC, Gallen IW, Smart CE, et al. Exercise management in type 1 diabetes: a consensus statement. Lancet Diabetes Endocrinol 2017;5:377-90.

13 Colberg SR, Sigal RJ, Yardley JE, et al. Physical Activity/Exercise and diabetes: a position statement of the American diabetes association. Diabetes Care 2016;39:2065-79.

14 Riddell MC, Zaharieva DP, Tansey M, et al. Individual glucose responses to prolonged moderate intensity aerobic exercise in adolescents with type 1 diabetes: the higher they start, the harder they fall. Pediatr Diabetes 2018;18:pedi.12799.

15 Siafarikas A, Johnston RJ, Bulsara MK, et al. Early loss of the glucagon response to hypoglycemia in adolescents with type 1 diabetes. Diabetes Care 2012;35:1757-62.

16 Dagogo-Jack SE, Craft S, Cryer PE. Hypoglycemia-Associated autonomic failure in insulin-dependent diabetes mellitus. recent antecedent hypoglycemia reduces autonomic responses to, symptoms of, and defense against subsequent hypoglycemia. $\mathrm{J}$. Clin. Invest. 1993;91:819-28.

17 Kishore P, Gabriely I, Cui M-H, et al. Role of hepatic glycogen breakdown in defective counterregulation of hypoglycemia in intensively treated type 1 diabetes. Diabetes 2006;55:659-66.

18 Bernroider E, Brehm A, Krssak M, et al. The role of intramyocellular lipids during hypoglycemia in patients with intensively treated type 1 diabetes. J Clin Endocrinol Metab 2005;90:5559-65.

19 Petersen KF, Price TB, Bergeron R. Regulation of net hepatic glycogenolysis and gluconeogenesis during exercise: impact of type 1 diabetes. J Clin Endocrinol Metab 2004;89:4656-64.

20 Cree-Green M, Newcomer BR, Brown MS, et al. Delayed skeletal muscle mitochondrial ADP recovery in youth with type 1 diabetes relates to muscle insulin resistance. Diabetes 2015;64:383-92.

21 Brugnara L, Vinaixa M, Murillo S, et al. Metabolomics approach for analyzing the effects of exercise in subjects with type 1 diabetes mellitus. PLoS One 2012;7:e40600.

22 Jenni S, Oetliker C, Allemann S, et al. Fuel metabolism during exercise in euglycaemia and hyperglycaemia in patients with type 1 diabetes mellitus--a prospective single-blinded randomised crossover trial. Diabetologia 2008;51:1457-65.

23 Bally L, Bovet C, Nakas CT, et al. A metabolomics approach to uncover effects of different exercise modalities in type 1 diabetes. Metabolomics 2017;13:1-5.

24 Battelino T, Danne T, Bergenstal RM, et al. Clinical targets for continuous glucose monitoring data interpretation: recommendations from the International consensus on time in range. Diabetes Care 2019;42:1593-603.

25 Jeukendrup AE, Wallis GA. Measurement of substrate oxidation during exercise by means of gas exchange measurements. Int $J$ Sports Med 2005;26 Suppl 1:S28-37.

26 Hackney AC, Lane AR. Exercise and the Regulation of Endocrine Hormones. In: Progress in molecular biology and translational science. . Elsevier B.V, 2015: 135. 293-311.

27 Galassetti P, Tate D, Neill RA, et al. Effect of differing antecedent hypoglycemia on counterregulatory responses to exercise in type 1 diabetes. Am J Physiol Endocrinol Metab 2006;290:E1109-17.

28 Schwartz NS, Clutter WE, Shah SD, et al. Glycemic thresholds for activation of glucose counterregulatory systems are higher than the threshold for symptoms. J Clin Invest 1987;79:777-81.

29 Seaquist ER, Anderson J, Childs B, et al. Hypoglycemia and diabetes: a report of a Workgroup of the American diabetes association and the endocrine Society. Diabetes Care 2013;36:1384-95

30 Workgroup on Hypoglycemia, American Diabetes Association. Defining and reporting hypoglycemia in diabetes: a report from the American diabetes association Workgroup on hypoglycemia. Diabetes Care 2005;28:1245-9.
31 Cryer P. Hierarchy of physiological responses to hypoglycemia: relevance to clinical hypoglycemia in type I (insulin dependent) diabetes Mellitus*. Horm Metab Res 1997;29:92-6.

32 Amiel SA, Sherwin RS, Simonson DC, et al. Effect of intensive insulin therapy on glycemic thresholds for counterregulatory hormone release. Diabetes 1988;37:901-7.

33 Bolli G, de Feo P, Compagnucci P, et al. Abnormal glucose counterregulation in insulin-dependent diabetes mellitus. interaction of anti-insulin antibodies and impaired glucagon and epinephrine secretion. Diabetes 1983;32:134-41.

34 Gosmanov NR, Szoke E, Israelian Z, et al. Role of the decrement in intraislet insulin for the glucagon response to hypoglycemia in humans. Diabetes Care 2005;28:1124-31.

35 Raju B, Cryer PE. Loss of the decrement in intraislet insulin plausibly explains loss of the glucagon response to hypoglycemia in insulindeficient diabetes: documentation of the intraislet insulin hypothesis in humans. Diabetes 2005;54:757-64.

36 Cooperberg BA, Cryer PE. Beta-cell-mediated signaling predominates over direct alpha-cell signaling in the regulation of glucagon secretion in humans. Diabetes Care 2009;32:2275-80.

37 Lindholm A, Jacobsen LV. Clinical pharmacokinetics and pharmacodynamics of insulin aspart. Clin Pharmacokinet 2001;40:641-59.

38 Vieira AF, Costa RR, Macedo RCO, et al. Effects of aerobic exercise performed in fasted $v$. fed state on fat and carbohydrate metabolism in adults: a systematic review and meta-analysis. Br J Nutr 2016;116:1153-64

39 Ball D. Metabolic and endocrine response to exercise: sympathoadrenal integration with skeletal muscle. J Endocrinol 2015;224:R79-95.

40 Sperling O. Human purine metabolism. Dordrecht: Springer, 1988: 225-36.

41 Sahlin K, Gorski J, Edström L. Influence of ATP turnover and metabolite changes on IMP formation and glycolysis in rat skeletal muscle. Am J Physiol 1990;259:C409-12.

42 Avila MA, García-Trevijano ER, SC L, et al. Molecules in focus methylthioadenosine. Int J Biochem Cell Biol 2004;36:2125-30.

43 Houten SM, Wanders RJA. A general introduction to the biochemistry of mitochondrial fatty acid $\beta$-oxidation. $J$ Inherit Metab Dis 2010;33:469-77.

44 Aquilano K, Baldelli S, Ciriolo MR. Glutathione: new roles in redox signaling for an old antioxidant. Front Pharmacol 2014:5:196.

45 Ceriello A, Novials A, Ortega E, et al. Glucagon-Like peptide 1 reduces endothelial dysfunction, inflammation, and oxidative stress induced by both hyperglycemia and hypoglycemia in type 1 diabetes. Diabetes Care 2013;36:2346-50.

46 Lewis GD, Farrell L, Wood MJ, et al. Metabolic signatures of exercise in human plasma. Sci Transl Med 2010;2:33ra37.

47 Steinhauser ML, Olenchock BA, O'Keefe J, et al. The circulating metabolome of human starvation. JCl Insight 2018;3.

48 Wolf M, Chen S, Zhao X, et al. Production and release of acylcarnitines by primary myotubes reflect the differences in fasting fat oxidation of the donors. J Clin Endocrinol Metab 2013;98:E1137-42

49 Fanelli CG, De Feo P, Porcellati F, et al. Adrenergic mechanisms contribute to the late phase of hypoglycemic glucose counterregulation in humans by stimulating lipolysis. J. Clin. Invest. 1992;89:2005-13.

50 Rattarasarn C, Dagogo-Jack S, Zachwieja JJ, et al. HypoglycemiaInduced autonomic failure in IDDM is specific for stimulus of hypoglycemia and is not attributable to prior autonomic activation. Diabetes 1994;43:809-18.

51 Cryer PE. Hypoglycemia-Associated autonomic failure in diabetes. Am J Physiol Endocrinol Metab 2001;281:E1115-21.

52 Sandoval DA, Aftab Guy DL, Richardson MA, et al. Acute, same-day effects of antecedent exercise on counterregulatory responses to subsequent hypoglycemia in type 1 diabetes mellitus. Am J Physio Endocrinol Metab 2006;290:E1331-8.

53 Sandoval DA, Guy DLA, Richardson MA, et al. Effects of low and moderate antecedent exercise on counterregulatory responses to subsequent hypoglycemia in type 1 diabetes. Diabetes 2004;53:1798-806. 